\title{
Stroke Secondary to Traumatic Carotid Artery Injury - A Case Report
}

\author{
Zoltán Bajkó1,2*, Smaranda Maier ${ }^{1,2}$, Anca Moțățăianu1,2, Rodica Bălașa ${ }^{1,2}$, Smaranda \\ Vasiu², Adina Stoian2, 3, Sebastian Andone² \\ ${ }^{1}$ Department of Neurology, University of Medicine and Pharmacy Târgu Mureș, Romania \\ 2 Mureș County Clinical Emergency Hospital, Târgu Mureș, Romania \\ ${ }^{3}$ Department of Pathophysiology, University of Medicine and Pharmacy Târgu Mureș, Romania
}

\begin{abstract}
Introduction: Lesions of the carotid and vertebral arteries secondary to direct trauma, called blunt cerebrovascular injuries (BCVI) are relatively rare and are markedly different from spontaneous dissections. Ischaemic stroke is a significant complication, with high morbidity and mortality rates. The basis of a diagnosis relies on appropriate, high sensitivity imaging screening.

Case report: We present the case of a 31 years old male patient with polytraumatism secondary to a motor vehicle accident, who was admitted to an orthopaedic clinic for multiple lower extremity fractures. His fractures were treated surgically. He developed in the 3rd day after the admission left sided hemiparesis secondary to ischaemic stroke. The diagnosis of traumatic carotid artery injury (TCAI) was based on duplex ultrasound and angio CT scans. The outcome was favourable despite the severe carotid lesions presenting with occlusion secondary to dissection.

Conclusions: In the majority of BCVI cases there is a variable latent period between the time of injury and the development of stroke. The management of cases is challenging because in the majority of cases there are multiple associated injuries. Although antithrombotics are widely used in the treatment, there is no consensus regarding the type of agent, the optimal dose or treatment duration.
\end{abstract}

Keywors: blunt cerebrovascular injuries, traumatic carotid artery injury, post-traumatic dissection, carotid artery, stroke

Received: 11 August 2017 / Accepted: 26 January 2018

\section{INTRODUCTION}

The term blunt cerebrovascular injury (BCVI) encompasses two distinct clinical entities: (a) traumatic carotid artery (TCAI) and (b) vertebral artery (TVAI) injuries. The reported incidence of these has increased owing to the extensive application of screening imagistic methods, such as ultrasound, CT angiography and digital subtraction angiography (DSA). $(1,2)$

These types of injuries are associated with morbidity and mortality rates ranging between 23 to $28 \%$, and $48-58 \%$ of survivors have significant neurological complications. $(3,4)$
Blunt cerebrovascular injuries differ in many ways from spontaneous carotid, and vertebral artery dissections and frequently are associated with other traumatic lesions. This complicates their management. Ischemic stroke appears in 10 to $-20 \%$ of patients with BCVI.(5) If unrecognised and untreated, the reported incidence increases to near $60 \%$. (6)

This case report aims to highlight the characteristics of a classic case of stroke secondary to TCAI and to discuss the literature data regarding the epidemiology, clinical presentation, diagnostic workup and treatment. 


\section{- CASE REPORT}

A 31 years old male patient sustained a poly-traumatic injury in a car accident. He was conscious when first seen at the accident site. The patient complained of pain localised to the lower extremities. He was transferred to the emergency unit of the Emergency County Hospital Tîrgu Mureş, Romania. Screening examinations evidenced fractures of the head of the left femur and right patellar. No other traumatic lesions were identified. There were no bruising or signs of seat belt impressions on his neck. Neurological deficits were not observed. Cerebral and cervical CT scans were undertaken without contrast agents. No abnormal conditions were identified. The patient was admitted to the Orthopaedic Clinic of the Emergency County Hospital Tîrgu Mureş, Romania, where his fractures were treated surgically. He was sedated, and painkillers were prescribed, tramadol $2 \times 100 \mathrm{mg}$ iv, fentanyl $0.1 \mathrm{mg}$ iv, metamizol 2x500 mg/zi iv.

Three days after admittance to the hospital the patient was neurologically asymptomatic. Then, on the fourth day, the patient awoke in the morning presenting with a left-sided hemiparesis. Neurological examination revealed left-sided central facial palsy and Grade 3 left-sided hemiparesis with diminished deep tendon reflexes and pyramidal signs.

An emergency cerebral CT scan and MRI revealed a right middle cerebral artery infarction. (Figure 1,2)

The duplex ultrasound examination of the cervical vessels revealed, at the proximal level of the right internal carotid artery (ICA), an intimal flap and a significant narrowing of the vessel lumen by a hypoechoic wall mass, suggestive for a mural hematoma. (Figure 3)

The cervical angio-CT scan confirmed the diagnosis of dissection of the right ICA, evidencing the suggestive tapering occlusion. (Figure 4)

Based on the imaging findings, a diagnosis of posttraumatic right ICA dissection was established. The mechanism of dissection, in this case, was not evident, though probably caused by direct pressure to the neck by the seatbelt or the hyperextension and contra-lateral rotation of the head which caused stretching of the ICA across the lateral processes of the cervical vertebra.

The patient was outside the time window for interventional therapy. He was transferred to the Neurology ward of the Emergency County Hospital Tîrgu Mureş, Romania. Anticoagulation therapy with a full dose of enoxaparine, $2 \times 0.8 \mathrm{ml} \mathrm{sc}$ was initiated and continued for two weeks. The patient neurological status stabilised. Rehabilitation therapy was started during the hospitalisation period. Two weeks after the onset of the stroke symptoms, the patient was discharged with Grade 4 hemiparesis. He was prescribed oral anticoagulation with vitamin $\mathrm{K}$ antagonist, acenocoumarol, 2 $\mathrm{mg} /$ day, with target INR range between 2 and 3 .

The patient continued with the rehabilitation therapy, after discharge. Complete remission of the hemiparesis was recorded at the neurological examination, one month after the discharge. At that time, the control duplex ultrasound examination showed lack of recanalization of the proximal ICA. Similar ultrasound findings were evidenced at a check-up examination, three months after the discharge.

\section{DISCUSSION}

Post-traumatic carotid artery dissections appear to occur more frequently than in vertebral arteries. (7)

Motor vehicle accidents are the main cause of BCVI, being responsible for more than $50 \%$. Other important causes are the assaults, falls, and attempted suicides. (8)

The main mechanism of blunt carotid artery injuries is the hyperextension and contra-lateral rotation of the head, as a result the ICA can be stretched across the lateral processes of the cervical vertebra. Another important mechanism is the direct pressure to the neck, e.g. by a seatbelt. The former was probably responsible for the carotid artery dissection in the present case. (9)

In the majority of BCVI cases, the pathological background is disruption to the intima, which causes luminal narrowing or even occlusion due to the collapse of the false lumen against the true lumen. The exposed sub-endothelial collagen promotes platelet aggregation and thrombus formation that can lead to embolization. This is the main mechanism of stroke following arterial dissection. Complete or partial trans-section can also occur, leading to pseudo-aneurysm formation. Another factor that can lead to a stroke in poly-traumatized patients is the hypercoagulable state, secondary to extensive tissue damage. (5)

The outer arterial wall in BCVI is usually unaffected. This occurs more frequently in case of spontaneous dissections.(5)

The clinical manifestations of BCVI are variable and depends upon the affected artery, injury site and grade 


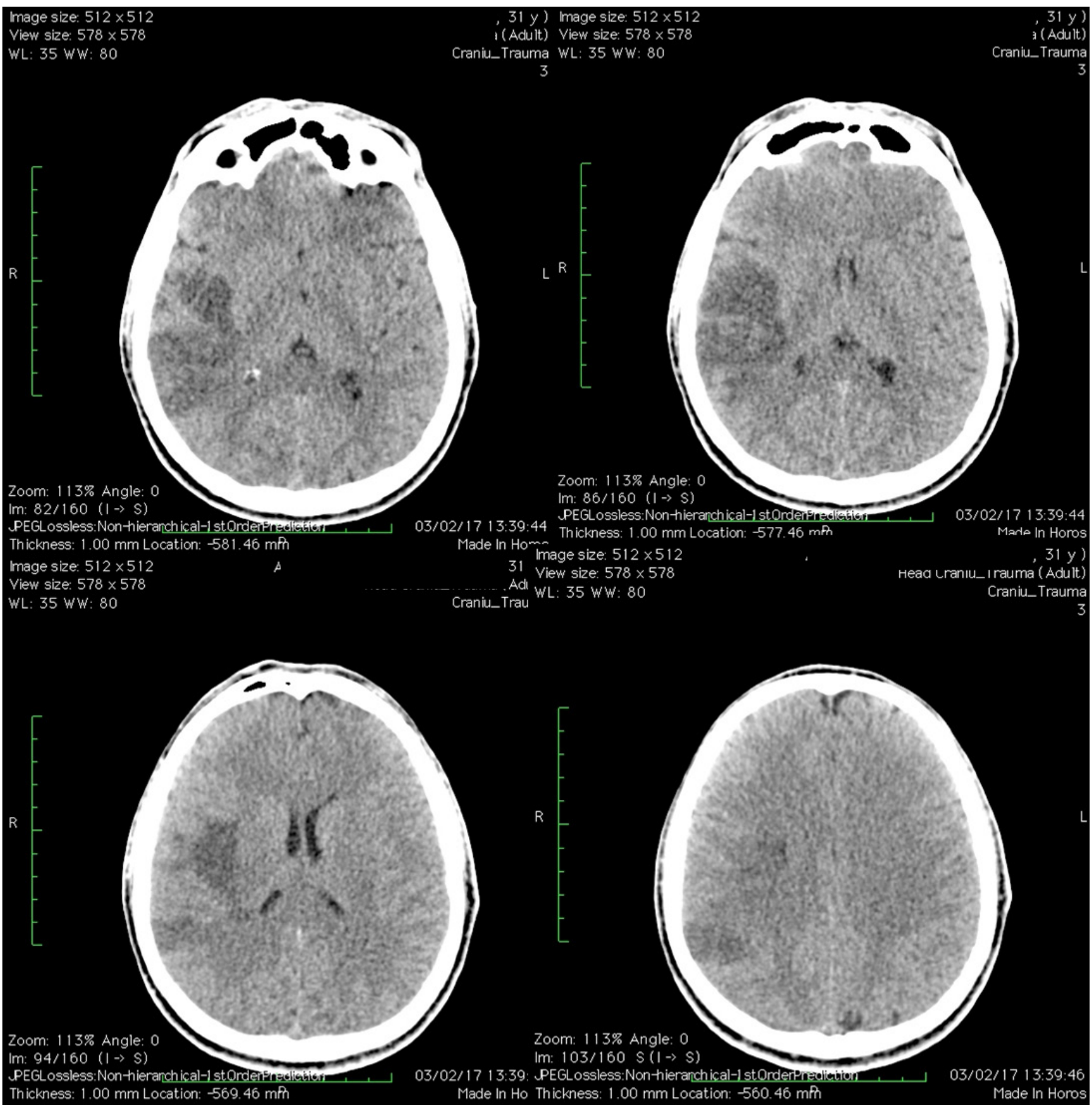

Fig. 1. Cerebral CT scan, axial sections revealing a right middle cerebral artery infarction

and the underlying clinical status of the patient. Usually, there is a latent period between the time of injury and the onset of the symptoms. The average time for a stroke to develop is between 12-75 hours post trauma, (10) although this may vary from several hours to weeks. $(11,12)$

The optimal screening criteria for BCVI of polytraumatized patients are debatable. The main risk factors for BCVI are listed as, a low Glasgow coma scale, petrous fractures, diffuse axonal injury and LeFort II and III fractures. However $20 \%$ of the patients with BCVI were seen not to have these risk factors. (13)

Cervical bruises would be a physical sign, indicative of screening for BCVI.(14. 15). In our case cervical bruising was absent, probably because of the complete occlusion of ICA. Jacobsen et al [ 2015] highlighted the limitations of relying only on risk factors to screen for BCVI, showing that an enhanced screening protocol (CTA) led to a five-fold increase in the identification of BCVI. Thirty percent of their patients were devoid of 


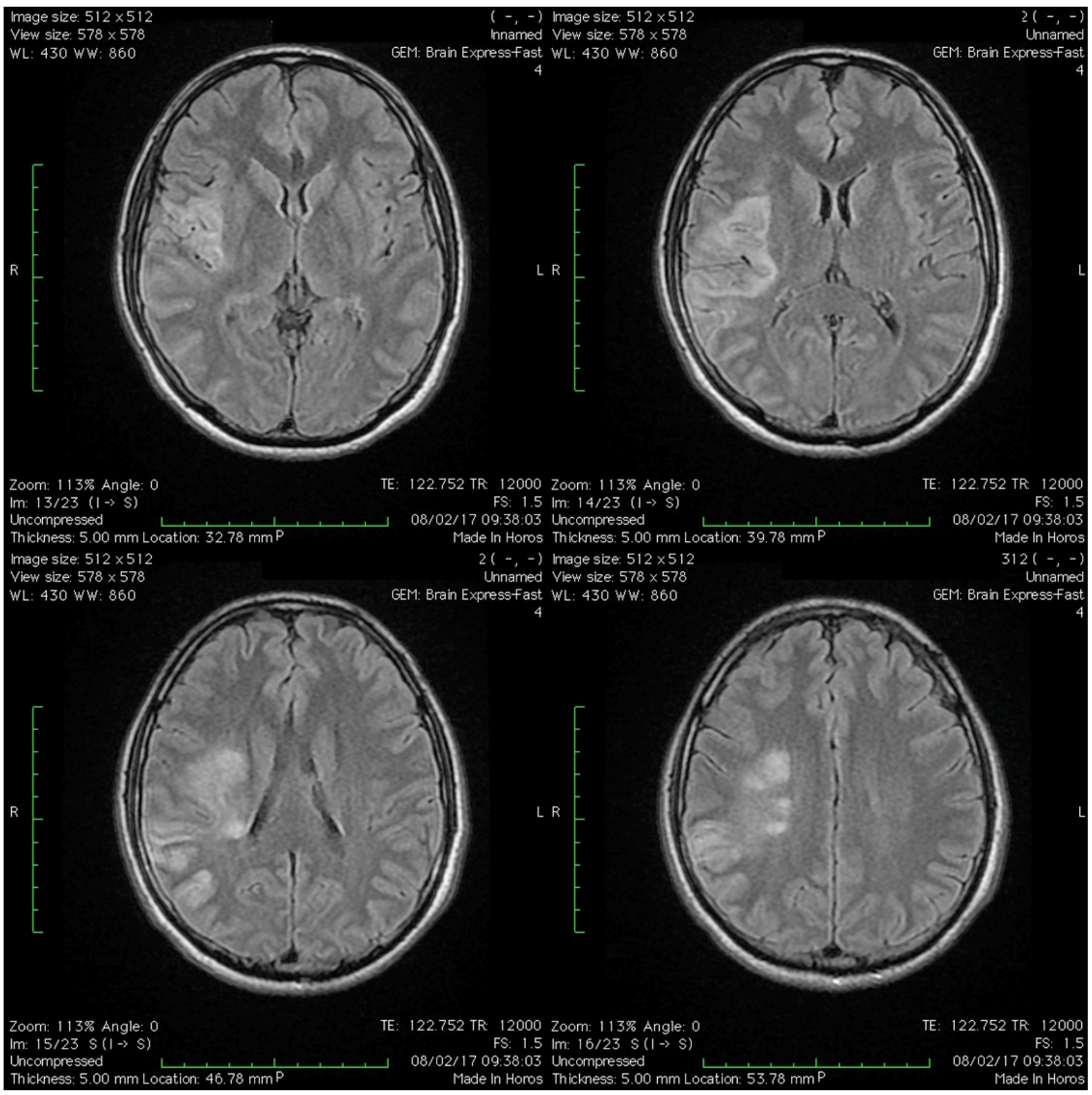

Fig. 2. T2-FLAIR weighted cerebral MRI, axial sections revealing a right middle cerebral artery infarction.

clinical signs, symptoms or risk factors. They detailed that CTAs of the neck were assessed several times, first by an attending neuroradiologist, then by an experienced CT radiologist who provide a preliminary reading. The following day, any positive or equivocal issues are reviewed by the attending neuroradiologist .(6) In our department, all scans are interpreted by a general radiologist. Jacobsen et al. [2015] promote a protocol that implies routine CTA of the neck in any blunt trauma patient who was already undergoing CT of the cervical spine. (6)
Digital subtraction angiography (DSA) is the gold standard for the diagnosis of BCVI but has been largely superseded by CT angiography. The latter is the screening test of choice in emergency conditions. Often, patients that require screening for BCVI also need a CT scan of other regions of the body, such as the spinal cord, chest and abdomen. $(5,13)$

CT devices with 16 or more slices have a high sensitivity and specificity for this type of injury. DSA is necessary when the results of other imaging methods are equivocal. $(5,13)$ 


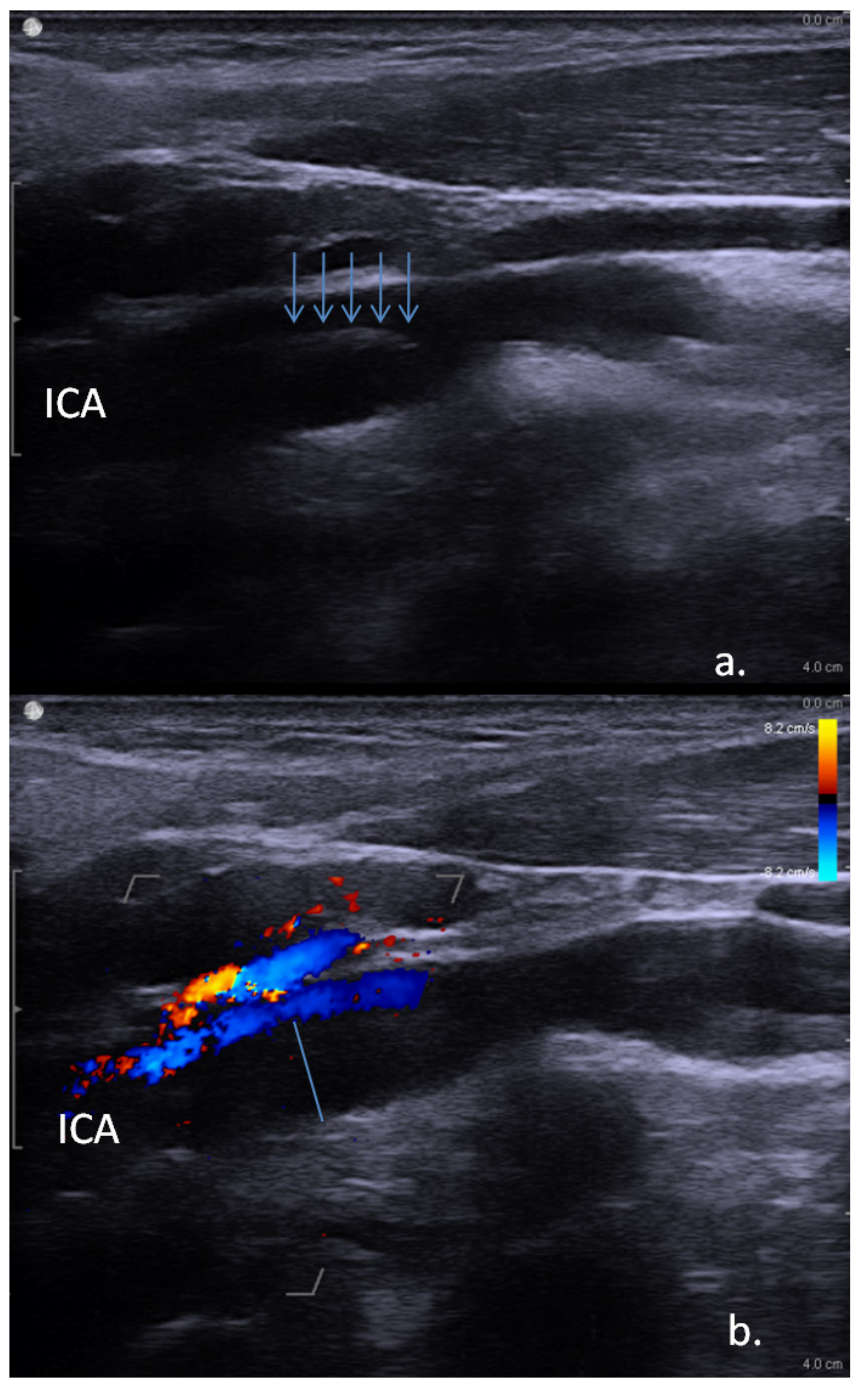

Fig. 3. Ultrasound examination of the right internal carotid artery. a. B-mode ultrasound examination revealing a white line in the vascular lumen (arrow) suggestive of an intimal flap; b. Colour mode ultrasound examination revealing a significant narrowing of the lumen secondary to the hypoechoic mural haematoma (blue line)

According to Grahndi R et al.[2017] a multi-detector CTA scan, as a screening tool for BCVI, has a false positive rate of $47.9 \%$. They promote DSA after positive findings on a CTA scan, in patients with suspected BCVI, which they claim, contributes to an increased diagnostic accuracy, thus diminishing the risk associated with unnecessary antithrombotic therapy. (16)

MR angiography is not widely available for screening and is time-consuming in emergency situations. (13)

Duplex ultrasonography (DU) is possible and noninvasive, but is sensitive only for the proximal and cervical portion of the vessels. Significant BCVI injuries

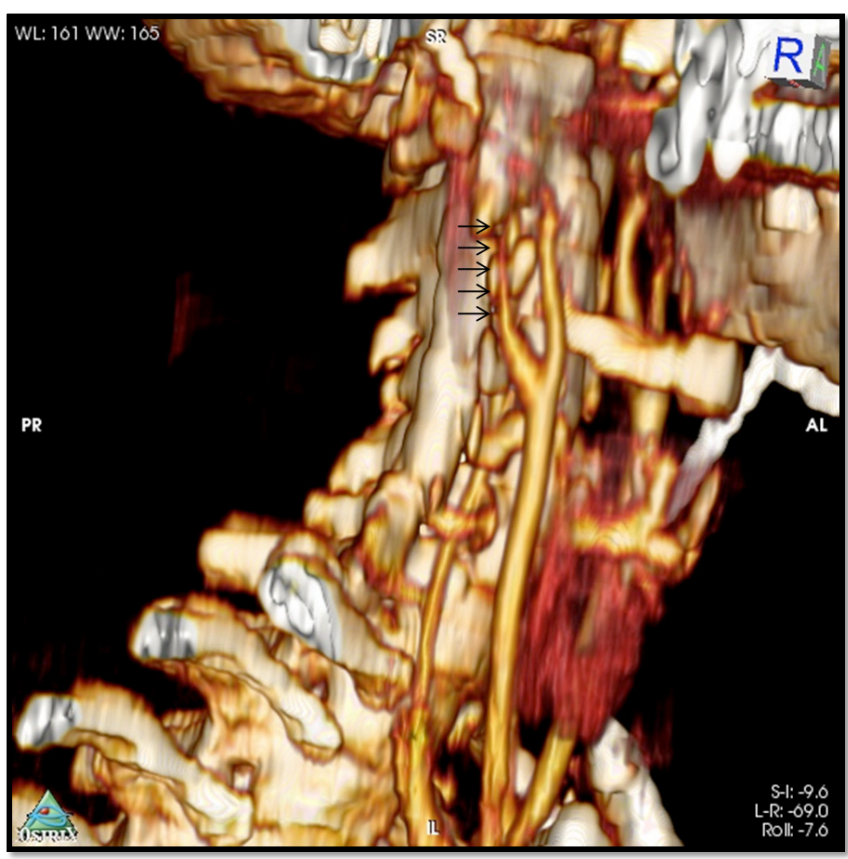

Fig. 4. Angio-CT examination of the cervical vessels revealing tapering occlusion, suggestive for ICA dissection.

occur at the base of the skull, and ultrasound imaging can provide only indirect signs of substantial distal injuries such as vessel occlusion or severe stenosis. As a result, DU has a limited role in the screening of selected cases. In our case, it was the basis for the diagnosis. (13)

According to a widely used classification scheme, BCVI can be divided into five subtypes: 1 . Luminal irregularity or dissection with $<25 \%$ stenosis; 2 . Dissection or intramural haematoma with $\geq 25 \%$ stenosis; 3 . Traumatic aneurysm; 4. Occlusion; 5 . Transection.

The present case was classified as subtype 4. (5)

Because of the low incidence, there are no evidencebased guidelines available for BCVI. The basis of the treatment is antithrombotic therapy. The optimal schedule regarding the medicaments, treatment duration and the end-point of the therapy is not known. Coexistent injuries contraindicate thrombolytic therapy in the majority of BCVI cases. Endovascular therapy such as mechanical thrombectomy or stenting can be an option in selected cases. (5)

Grade 4 injuries are associated with high morbidity and mortality, and the outcome correlates with the severity of neurologic impairment on presentation.(8) In our case the result was excellent.

The recanalisation rate of Grade 4 injuries is low. In these cases, it is useful to initiate antithrombotic treatment with anticoagulants, if there is no contraindication, followed by long-term antiplatelet therapy (13). It 
is believed that initiation of antiplatelet or anticoagulation during the symptom-free interval might reduce the stroke rate to below $1 \%$. (11-17)

\section{- CONCLUSIONS}

Blunt cerebro-vascular injuries are rare, but potentially life-threatening conditions that require prompt diagnosis and management. Screening investigations, such as angio-CT scans are indicated in high-risk patients. Duplex ultrasound can be useful in selected cases. There is, in the majority of cases, a variable latent period between the time of injury and the occurrence of a symptomatic stroke. Although antithrombotics are widely used in the treatment, there are no guidelines regarding the type of agent, the proper dose or treatment duration.

\section{CONFLICT OF INTEREST}

None to declare.

\section{DEFERENCES}

1. Liang T, Tso DK, Chiu RY, Nicolaou S. Imaging of blunt vascular neck injuries: a clinical perspective. Am J Roentgenol. 2013;201:893901.

2. Langner S, Fleck S, Kirsch M, Petrik M, Hosten N. Whole-body $\mathrm{CT}$ trauma imaging with adapted and optimized CT angiography of the craniocervical vessels: do we need an extra screening examination? Am J Neuroradiol. 2008;29:1902-7.

3. Biffl WL, Moore EE, Ryu RK, et al. The unrecognized epidemic of blunt carotid arterial injuries: early diagnosis improves neurologic outcome. Ann Surg. 1998;228:462-70.

4. Bajkó Z, Bălaşa R, Moţăţăianu A, et al. Malignant Middle Cerebral Artery Infarction Secondary to Traumatic Bilateral Internal Carotid Artery Dissection. A Case Report. J Crit Care Med. 2016;2:135-41.
5. Foreman PM, Harrigan MR. Blunt Traumatic Extracranial Cerebrovascular Injury and Ischemic Stroke. Cerebrovasc Dis Extra. 2017;7:72-83.

6. Jacobson EL, Ziemba-Davis M, Herrera AJ. The limitations of using risk factors to screen for blunt cerebrovascular injuries: the harder you look, the more you find. World J Emerg Surg. 2015;10:46.

7. Biffl WL, Ray CE Jr, Moore EE, et al. Treatment related outcomes from blunt cerebrovascular injuries: importance of routine followup arteriography. Ann Surg. 2002;235:699-706.

8. Cogbill TH, Moore EE, Meissner M, et al. The spectrum of blunt injury to the carotid artery: a multicenter perspective. J Trauma. 1994;37:473-9.

9. Zelenock GB, Kazmers A, Whitehouse WM Jr, et al. Extracranial internal carotid artery dissections: noniatrogenic traumatic lesions. Arch Surg. 1982;117:425-32.

10. Fabian TC, George SM Jr, Croce MA, et al. Carotid artery trauma: management based on mechanism of injury. J Trauma. 1990;30:953-61.

11. Cothren CC, Biffl WL, Moore EE, et al. Treatment for blunt cerebrovascular injuries equivalence of anticoagulation and antiplatelet agents. Arch Surg. 2009;144:685-90.

12. Ahmad HA, Gerraty RP, Davis SM, Cameron PA.Cervicocerebral artery dissections. J Accid Emerg Med. 1999;16:422-4.

13. Biffl WL, Burlew CC, Moore EE. Blunt cerebrovascular injury: Mechanisms, screening, and diagnostic evaluation. In: UpToDate, Collins KA (ED), UpToDate, Waltham, MA. (Accessed on May 13, 2017.)

14. Bromberg WJ, Collier BC, Diebel LN, et al. Blunt cerebrovascular injury practice management guidelines: The eastern association for the surgery of trauma. J Trauma. 2010;68:471-77.

15. Biffl WL, Moore EE, Offner PJ, et al. Optimizing screening for blunt cerebrovascular injuries. Am J Surg. 1999;178:517-22.

16. Grandhi R, Weiner GM, Agarwal N, et al. Limitations of multidetector computed thomography angiography for the diagnosis of blunt cerebrovascular injury. J Neurosurg. 2017;11:1-6.

17. Burlew CC, Biffl WL. Blunt cerebrovascular trauma. Curr Opin Crit Care. 2010;16:587-95. 